\title{
On the Cesáro Summability of Double Series
}

\author{
E. Savaş, ${ }^{1}$ H. Şevli, ${ }^{2}$ and B. E. Rhoades ${ }^{3}$ \\ ${ }^{1}$ Department of Mathematics, Istanbul Commerce University, 34672 Üsküdar, Istanbul, Turkey \\ ${ }^{2}$ Department of Mathematics, Faculty of Arts E Sciences, Yüzüncü Yil University, 65080 Van, Turkey \\ ${ }^{3}$ Department of Mathematics, Indiana University, Bloomington, IN 47405, USA
}

Correspondence should be addressed to E. Savaş, ekremsavas@yahoo.com

Received 18 July 2007; Accepted 19 August 2007

Recommended by Martin J. Bohner

In a recent paper by Savaş and Şevli (2007), it was shown that each Cesáro matrix of order $\alpha$, for $\alpha>-1$, is absolutely $k$ th power conservative for $k \geq 1$. In this paper we extend this result to double Cesáro matrices.

Copyright $(02008$ E. Savaş et al. This is an open access article distributed under the Creative Commons Attribution License, which permits unrestricted use, distribution, and reproduction in any medium, provided the original work is properly cited.

The concept of absolute summability of order $k \geq 1$ was defined by Flett [1] as follows. Let $\sum a_{k}$ be a series with partial sums $\left(s_{n}\right), A$ an infinite matrix. Then $\sum a_{k}$ is said to be absolutely summable $A$ of order $k \geq 1$ if

$$
\sum_{n=1}^{\infty} n^{k-1}\left|T_{n-1}-T_{n}\right|^{k}<\infty
$$

where

$$
T_{n}:=\sum_{k=0}^{\infty} a_{n k} s_{k}
$$

Denote by $\mathcal{A}_{k}$ the sequence space defined by

$$
\mathscr{A}_{k}=\left\{\left(s_{n}\right): \sum_{n=1}^{\infty} n^{k-1}\left|a_{n}\right|^{k}<\infty ; a_{n}=s_{n}-s_{n-1}\right\}
$$

for $k \geq 1$. A matrix $T$ is said to be a bounded linear operator on $\mathscr{A}_{k}$, written $T \in B\left(\mathcal{A}_{k}\right)$, if $T: \mathscr{A}_{k} \rightarrow \mathscr{A}_{k}$. In 1970, Das [2] defined such a matrix to be absolutely $k$ th power conservative 
for $k \geq 1$. In that paper, he proved that every conservative Hausdorff matrix $H \in B\left(\mathcal{A}_{k}\right)$ for $k \geq 1$. In a recent paper [3], the first two authors proved every Cesáro matrix of order $\alpha$, for $\alpha>-1,(C, \alpha) \in B\left(\mathcal{A}_{k}\right)$ for $k \geq 1$. Since the Cesáro matrices of order $\alpha$ for $-1<\alpha<0$ are not conservative, their result shows that being conservative is not a necessary condition for being absolutely $k$ th power conservative.

In this paper, we extend the result of [3] to double summability, thereby demonstrating that the property of being conservative is again not necessary for doubly infinite matrices to be absolutely $k$ th power conservative.

Let $\sum_{m=0}^{\infty} \sum_{n=0}^{\infty} a_{m n}$ be an infinite double series with real or complex numbers, with partial sums

$$
s_{m n}=\sum_{i=0}^{m} \sum_{j=0}^{n} a_{i j}
$$

For any double sequence $\left(x_{m n}\right)$, we will define

$$
\Delta_{11} x_{m n}=x_{m n}-x_{m+1, n}-x_{m, n+1}+x_{m+1, n+1} .
$$

The series $\sum \sum a_{m n}$ is said to be summable $|C, \alpha, \beta|_{k^{\prime}}, k \geq 1, \alpha, \beta>-1$, if (see [4])

$$
\sum_{m=1}^{\infty} \sum_{n=1}^{\infty}(m n)^{k-1}\left|\Delta_{11} \sigma_{m-1, n-1}^{\alpha \beta}\right|^{k}<\infty
$$

where $\sigma_{m n}^{\alpha \beta}$ denotes the $m n$-term of the $(C, \alpha, \beta)$ transform of a sequence $\left(s_{m n}\right)$, that is,

$$
\sigma_{m n}^{\alpha \beta}=\frac{1}{E_{m}^{\alpha} E_{n}^{\beta}} \sum_{i=0}^{m} \sum_{j=0}^{n} E_{m-i}^{\alpha-1} E_{n-j}^{\beta-1} s_{i j} .
$$

Define

$$
\mathcal{A}_{k}^{2}:=\left\{\left(s_{m n}\right)_{m, n=0}^{\infty}: \sum_{m=1}^{\infty} \sum_{n=1}^{\infty}(m n)^{k-1}\left|a_{m n}\right|^{k}<\infty ; a_{m n}=\Delta_{11} s_{m-1, n-1}\right\}
$$

for $k \geq 1$.

A four-dimensional matrix $T=\left(t_{m n i j}: m, n, i, j=0,1, \ldots\right)$ is said to be absolutely $k$ th power conservative, for $k \geq 1$, if $T \in B\left(\mathcal{A}_{k}^{2}\right)$; that is, if

$$
\sum_{m=1}^{\infty} \sum_{n=1}^{\infty}(m n)^{k-1}\left|\Delta_{11} s_{m-1, n-1}\right|^{k}<\infty
$$

implies that

$$
\sum_{m=1}^{\infty} \sum_{n=1}^{\infty}(m n)^{k-1}\left|\Delta_{11} t_{m-1, n-1}\right|^{k}<\infty
$$

where

$$
t_{m n}=\sum_{i=0}^{\infty} \sum_{j=0}^{\infty} t_{m n i j} s_{i j} \quad(m, n=0,1, \ldots) .
$$


Theorem 1. $(C, \alpha, \beta) \in B\left(\mathcal{A}_{k}^{2}\right)$ for each $\alpha, \beta>-1$.

Proof. Let $\tau_{m n}^{\alpha \beta}$ denote the $m n$-term of the $(C, \alpha, \beta)$-transform, in terms of $\left(m n a_{m n}\right)$; that is,

$$
\tau_{m n}^{\alpha \beta}=\frac{1}{E_{m}^{\alpha} E_{n}^{\beta}} \sum_{i=1}^{m} \sum_{j=1}^{n} E_{m-i}^{\alpha-1} E_{n-j}^{\beta-1} i j a_{i j} .
$$

For $\alpha, \beta>-1$, since

$$
\tau_{m n}^{\alpha \beta}=m n\left(\sigma_{m n}^{\alpha \beta}-\sigma_{m, n-1}^{\alpha \beta}-\sigma_{m-1, n}^{\alpha \beta}+\sigma_{m-1, n-1}^{\alpha \beta}\right),
$$

to prove the theorem, it will be sufficient to show that

$$
\sum_{m=1}^{\infty} \sum_{n=1}^{\infty} \frac{1}{m n}\left|\tau_{m n}^{\alpha \beta}\right|^{k}<\infty
$$

Using Hölder's inequality, we have

$$
\begin{aligned}
\sum_{m=1}^{\infty} \sum_{n=1}^{\infty} \frac{1}{m n}\left|\tau_{m n}^{\alpha \beta}\right|^{k} & =\sum_{m=1}^{\infty} \sum_{n=1}^{\infty} \frac{1}{m n}\left|\frac{1}{E_{m}^{\alpha} E_{n}^{\beta}} \sum_{i=1}^{m} \sum_{j=1}^{n} E_{m-i}^{\alpha-1} E_{n-j}^{\beta-1} i j a_{i j}\right|^{k} \\
& \leq \sum_{m=1}^{\infty} \sum_{n=1}^{\infty} \frac{1}{m n E_{m}^{\alpha} E_{n}^{\beta}} \sum_{i=1}^{m} \sum_{j=1}^{n} E_{m-i}^{\alpha-1} E_{n-j}^{\beta-1}(i j)^{k}\left|a_{i j}\right|^{k} \times\left\{\frac{1}{E_{m}^{\alpha} E_{n}^{\beta}} \sum_{i=1}^{m} \sum_{j=1}^{n} E_{m-i}^{\alpha-1} E_{n-j}^{\beta-1}\right\}^{k-1} .
\end{aligned}
$$

Since

$$
\frac{1}{E_{m}^{\alpha} E_{n}^{\beta}} \sum_{i=1}^{m} \sum_{j=1}^{n} E_{m-i}^{\alpha-1} E_{n-j}^{\beta-1}=1
$$

we obtain

$$
\begin{aligned}
\sum_{m=1}^{\infty} \sum_{n=1}^{\infty} \frac{1}{m n}\left|\tau_{m n}^{\alpha \beta}\right|^{k} & \leq \sum_{m=1}^{\infty} \sum_{n=1}^{\infty} \frac{1}{m n E_{m}^{\alpha} E_{n}^{\beta}} \sum_{i=1}^{m} \sum_{j=1}^{n} E_{m-i}^{\alpha-1} E_{n-j}^{\beta-1}(i j)^{k}\left|a_{i j}\right|^{k} \\
& \leq \sum_{i=1}^{\infty} \sum_{j=1}^{\infty}(i j)^{k}\left|a_{i j}\right|^{k} \sum_{m=i}^{\infty} \sum_{n=j}^{\infty} \frac{E_{m-i}^{\alpha-1} E_{n-j}^{\beta-1}}{m n E_{m}^{\alpha} E_{n}^{\beta}} .
\end{aligned}
$$

For $\alpha, \beta>-1$ and $m, n \geq 1$,

$$
\sum_{m=i}^{\infty} \sum_{n=j}^{\infty} \frac{E_{m-i}^{\alpha-1} E_{n-j}^{\beta-1}}{m n E_{m}^{\alpha} E_{n}^{\beta}}=\sum_{m=i}^{\infty} \frac{E_{m-i}^{\alpha-1}}{m E_{m}^{\alpha}} \sum_{n=j}^{\infty} \frac{E_{n-j}^{\beta-1}}{n E_{n}^{\beta}}=\frac{1}{j} \sum_{m=i}^{\infty} \frac{E_{m-i}^{\alpha-1}}{m E_{m}^{\alpha}}=(i j)^{-1}
$$

Thus

$$
\sum_{m=1}^{\infty} \sum_{n=1}^{\infty} \frac{1}{m n}\left|\tau_{m n}^{\alpha \beta}\right|^{k}=O(1) \sum_{i=1}^{\infty} \sum_{j=1}^{\infty}(i j)^{k}\left|a_{i j}\right|^{k} \frac{1}{i j}=O(1) \sum_{i=1}^{\infty} \sum_{j=1}^{\infty}(i j)^{k-1}\left|a_{i j}\right|^{k}=O(1)
$$

since $\left(s_{m n}\right) \in \mathcal{A}_{k}^{2}$. 
Using the notation of [5],

$$
\begin{aligned}
\theta_{m n}^{\alpha} & :=\frac{1}{E_{m}^{\alpha}} \sum_{i=0}^{m} E_{m-i}^{\alpha-1} s_{i n}=(C, \alpha, 0)\left(s_{m n}\right), \\
\theta_{m n}^{\beta} & :=\frac{1}{E_{n}^{\beta}} \sum_{j=0}^{n} E_{n-j}^{\beta-1} s_{m j}=(C, 0, \beta)\left(s_{m n}\right), \\
\sigma_{m n} & :=\frac{1}{(m+1)(n+1)} \sum_{i=0}^{m} \sum_{j=0}^{n} s_{i j}=(C, 1,1)\left(s_{m n}\right) .
\end{aligned}
$$

Corollary 1. $(C, \alpha, 0) \in B\left(\mathcal{A}_{k}^{2}\right)$ for each $\alpha>-1$.

Corollary 2. $(C, 0, \beta) \in B\left(\mathcal{A}_{k}^{2}\right)$ for each $\alpha>-1$.

Corollary 3. $(C, 1,1) \in B\left(\mathcal{A}_{k}^{2}\right)$.

\section{References}

[1] T. M. Flett, "On an extension of absolute summability and some theorems of Littlewood and Paley," Proceedings of the London Mathematical Society, vol. 7, pp. 113-141, 1957.

[2] G. Das, "A Tauberian theorem for absolute summability," Mathematical Proceedings of the Cambridge Philosophical Society, vol. 67, pp. 321-326, 1970.

[3] E. Savaş and H. Şevli, "On extension of a result of Flett for Cesáro matrices," Applied Mathematics Letters, vol. 20, no. 4, pp. 476-478, 2007.

[4] B. E. Rhoades, "Absolute comparison theorems for double weighted mean and double Cesàro means," Mathematica Slovaca, vol. 48, no. 3, pp. 285-301, 1998.

[5] M. Y. Mirza and B. Thorpe, "Tauberian constants for double series," Journal of the London Mathematical Society, vol. 57, no. 1, pp. 170-182, 1998. 